\title{
Big Data and Population Processes: A Revolution?
}

\section{Published in:}

Alessandra Petrucci, Rosanna Verde (edited by), SIS 2017. Statistics and Data Science: new challenges, new generations. 28-30 June 2017 Florence (Italy). Proceedings of the Conference of the Italian Statistical Society, Firenze University Press, 2017, pp. 167-178, CC BY 4.0.

Link to the E-Book

Francesco C. Billari and Emilio Zagheni

\begin{abstract}
We first discuss the centrality of data paradigms in demography, documenting their rise and fall over time also making use of Google Books Ngram Viewer. We then move on to discuss the undergoing "Data Revolution" in demography, with a focus on emerging forms of big data access and on the use of digital breadcrumbs.
\end{abstract}

\section{Four demographic data paradigms?}

In Kuhn's well-known discussion of the role of paradigms and "normal science" in scientific progress, the "normal" data to be used within a group of scholars are central to a paradigm. Discussions and debates take place in relation when paradigms are challenged: "The pre-paradigm period, in particular, is regularly marked by frequent and deep debates over legitimate methods, problems, and standards of solution" [19]. Given the data-intensive nature of demography, we characterize paradigms in demography by referring to the "normal" data used within a given paradigm.

Francesco C. Billari

Department of Policy Analysis and Public Management; Carlo F. Dondena Centre for Research on Social Dynamics and Public Policies; Bocconi Institute for Data Science and Analytics; Università Bocconi via Röntgen 1, 20136 Milano, Italy, e-mail: francesco.billari@unibocconi. it

Emilio Zagheni

Department of Sociology, University of Washington, 211 Savery Hall, Box 353340, Seattle, WA 98195-3340, USA, e-mail: emilioz@uw. edu 
We now illustrate four data paradigms in demography ${ }^{1}$. In order to illustrate the rise and fall of data paradigms we use the approach based on the prevalence of combination of terms (Ngrams) in books indexed in the Google corpus and accessible through Google Books Ngram Viewer ${ }^{2}$. [2].

\subsection{Census and administrative records}

That the study of population processes needs "Big Data" should come as no surprise. Indeed, data on population processes have always been "Big", relatively to the epoch. It is useful to shortly recall here instances from the history of population research, taking into account that, historically, governments, churches and local authorities were the monopolists of data collection, curation and storage: census and administrative records are the paradigmatic data in this first demographic data paradigm.

In addition to Malthus' ground-breaking work on the relationship between population change and economic development, which has been linked to the emergence of the modern population census, demographic research originates historically from the creative and innovative use of data originally collected for other purposes. Graunt's 1662 Natural and Political Observations Made upon the Bills of Mortality are considered the founding essay for demography, as well as for epidemiology and statistics. The patient and pioneering analysis of the bills, which were published at a weekly rate, together with the low technological level then available, tells us that Graunt's population data were already "Big", relative to the epoch.

In historical demography, Henry and his colleagues pioneered the systematic linkages of parish registers to reconstruct population processes. They started from a village to extending this reconstruction to broader geographical areas, and generalized the effort through the careful preparation and analysis of linked data [9]. The family reconstructions of Henry and colleagues were again already "Big data" for the epoch.

The use of population-wide individual-level register data has become the marker, and the comparative advantage of Nordic demographers, with later efforts to link individual-level Census records and other registers extending to other countries such as Belgium and the Netherlands in particular. Register data with individual identifiers (PINs, i.e. Personal Identification Numbers) that allow to link multiple individuals and to follow individuals over time are "Big Data". Systems of PINs have

\footnotetext{
${ }^{1}$ This section is loosely inspired by Gray's idea of a fourth paradigm [15]. Our characterization of four paradigms in demography differs from the one of Courgeau and Frank, who refer to the micro-macro perspective through which relationships between the individual- and population-level have been seen over time [6].

2 https://books.google.com/ngrams. Figures 1, 2, 3 and were generated using Google Books Ngram Viewer with the English language 2012 corpus, using data between 1800 and 2008. The robustness of results was checked against case sensitiveness. Héran [14] carried a detailed analysis of the "demographic vocabulary" using the same approach
} 
been implemented in Sweden in 1947, in Norway in 1961, in Finland in 1964, and in Denmark in 1968 [22]. This lead, after some time, to the abolition of population censuses, with register-based "Big Data" also replacing the census for population counts.

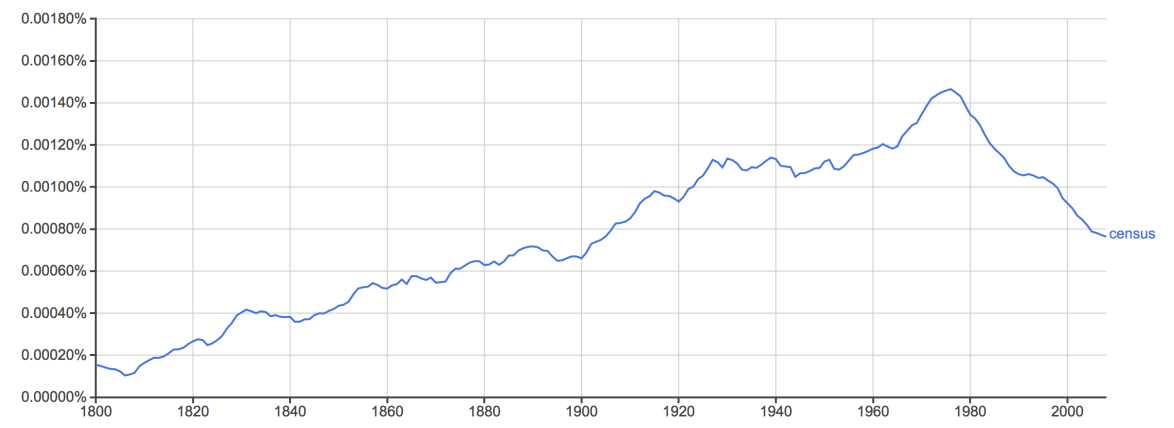

Fig. 1 The rise and fall of the "Census" in English books indexed in Google books. Source: https://books.google.com/ngrams

The demographic data paradigm based on census and administrative records is interested only in macro-level outcomes. Even when individual-level data are used as the starting point, the main interest is to quantify population-level parameters. Formal demography has emerged, developing the mathematical basis of the measurement of population-level quantities and of the study population dynamics, to complement and to inspire data analyses. To quantify the rise - and fall - of this paradigm in a graph, we here consider the emergence of the "census" as referred in books is depicted in Figure 1, where the peak is in the mid-1970s. ${ }^{3}$

\subsection{Theory-driven micro-level data}

After World War II, sample surveys began to be widespread to study population processes, following up on earlier development during the 1930s. During this period, "Demographers at the Bureau of Census, in collaboration with applied statisticians, began to develop sampling methods for meeting demands for timely measures of unemployment levels" [29]. By the end of the 1950s, at least in the United States, sample surveys have become central in social science research, including population research. By the end of the 1960s statistical packages have become available to analyze what were the "big Data" of that epoch. Theory-driven micro-level data are the paradigmatic ones in this second demographic data paradigm.

The World Fertility Survey (WFS), coordinated by the London office of the International Statistical Institute (ISI), is the first major attempt to field a comparable

\footnotetext{
${ }^{3}$ A similar trend over time could be found when restricting the search to "population census".
} 
sample survey across a wide range of countries, with 61 countries fielding a WFS between 1973 and 1984. At the outset of the programme, Sprehe, on behalf of the ISI states the basic aim of the WFS: "to provide scientific information that will permit each participating country to describe and interpret its population's fertility" [32]. All WFS have to include "independent variables" that measure factors affecting fertility, to be included in micro-level statistical analyses. The choice of these factors is based on existing fertility theories, and builds on earlier survey-based research.

Since the WFS, sample surveys have become a prime, if not the major, source for population research during the last quarter of the Twentieth Century, in particular for what concerns family and fertility research. Demographers have also engineered, through formal demography, ways to exploit limited and defective data in order to estimate population-level parameters from information available through, for instance, the Demographic and Health Surveys (DHS), the successor of the WFS for developing countries. This "demographic estimation" approach has been recently and systematically illustrated by Moultrie and colleagues [24].

While traditional formal demography remains significantly anchored at the macrolevel within this paradigm, there is a parallel development in which micro-level outcomes become the target of demographic research. The emergence of micro-level data, and of micro-level outcomes, as a central target in the study of population processes, and therefore to the second data paradigm in demography, is linked to the role of how micro- and meso-level factors influence demographic choices. Statistics comes to support this micro-level focus, and the 1972 article by David Cox [7] provides an elegant and general regression-based approach to life-table, three centuries after Graunt.

To quantify the rise - and fall - of this second paradigm, we look at two trends. First, the presence of the ngram "life table" or "life tables" as compared to "proportional hazards" in Figure 2. By the early 2000s "proportional hazards" basically reached the frequency of "life table" and seems to have started its decline. Second, in Figure 3, we show the rise and fall of the WFS as compared to the DHS and the Fertility and Family Survey between 1970 and 2008.

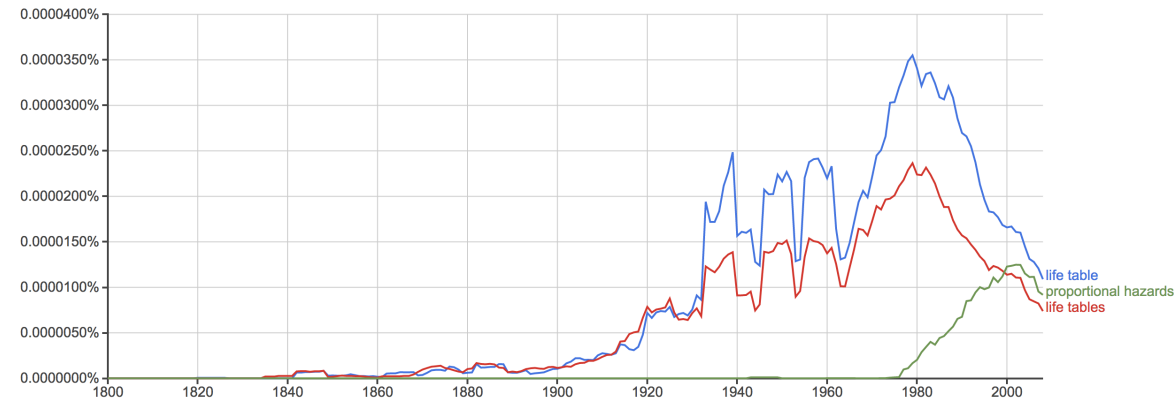

Fig. 2 Ngram prevalence for "life table" and of "proportional hazards" in English books indexed in Google books. Source: https://books.google.com/ngrams 


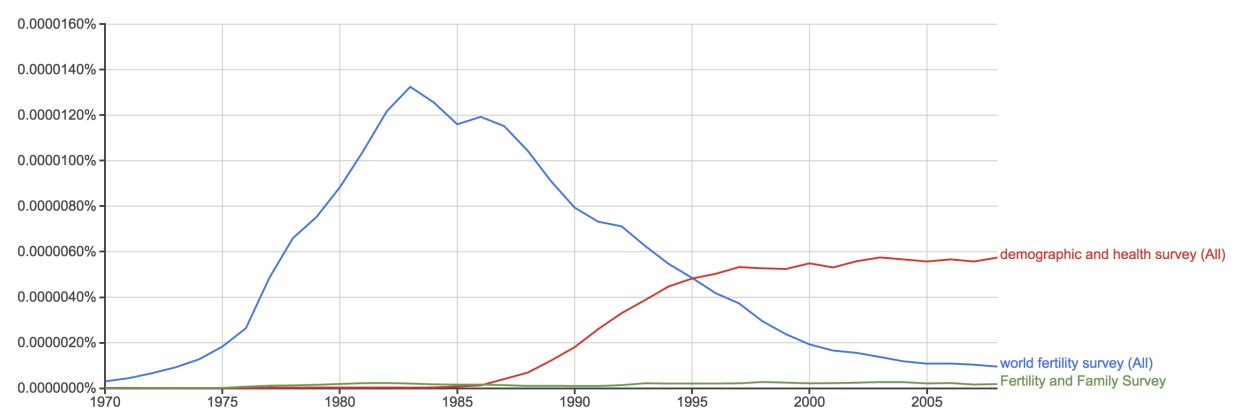

Fig. 3 Ngram prevalence for "World Fertility Survey", "Demographic and Health Survey", and "Fertility and Family Survey" in English books indexed in Google books. Source: https: // books.google.com/ngrams

\subsection{Data-driven discovery meets theory-driven discovery}

The key critique to the second demographic data paradigm, is that it leads to forget population-level processes, the ultimate object of population scholars [21]. The idea that a multi-level paradigm should substitute the micro-based one has been suggested, among others, by Courgeau and Frank [6].

One way to see the link between demography targeting macro- and micro-level outcomes, as well as the link between data and theory in population research, is to cast a two-stage view of demographic research, distinguishing a discovery stage from an explanation stage [5]. The first-discovery-stage aims at the production of novel evidence at the population level. While description is often abhorred in the social sciences, research on population processes has shown that it is fundamental to anchor science to solid empirical bases. However, only novel evidence contributes to the cumulation of knowledge. The second-explanation- stage aims at developing accounts of demographic change and tests how the action and interaction of individuals generate what is discovered in the first stage.

The distinction between discovery and explanation does not refer to the fact that discovery on population processes should only be data-driven. However, discovery should not only only be theory-driven, as for instance advocated by some social theorists [35] who do not give empirical discoveries a proper role in social science. The meeting between data-driven and theory-driven discovery has emerged in population research more recently, with a marriage between demography's "powerful descriptive potential" and causal analysis [25]. A data example of this meeting is the effort to link administrative records with theory-based survey data. The Generations and Gender Survey, for instance, in Nordic countries has exploited the available administrative records and linked them with the theory-based questionnaire [36].

In terms of methods, the key challenge for this third demographic data paradigm has been to link micro-level (data and theory) processes with macro-level population processes. The spread of computational, agent-based modeling has been seen as a potential solution. It is however too early to say whether this approach has made it to 
the core of a paradigm [2]. The systematic approach linking data- and theory-driven micro-founded simulation models with data at the population level has however become visible on demographic journals [3][17].

\subsection{A fourth paradigm?}

Are we at the dawn of a fourth demographic data paradigm? Yes and no: we are observing debates and trends that are typical of pre-paradigm shifts. The outcome is yet to be determined, but we identify three key crossroads.

Centralization vs Decentralization. Data collection and data interpretation, the essence of research, have been so far in the hands of a small minority of experts. Internet and the digital revolution have marked a discontinuity in practices of research. Everyone can potentially collect data for their own use or for research purposes, in various forms that include collecting genealogical family trees or using a mobile phone app to monitor health. The process is completely decentralized. However, corporations have emerged to tap into these new sources and bring them to a centralized repository. Similarly, the "open science" movement has brought nonprofessionals into the realm of research. Wikipedia is an example of a revolutionary form of mass-collaboration that goes beyond professional scientists to produce knowledge.

Bias vs Variance. Against the backdrop of decreasing survey response rates and the increasing availability of non-representative data, we are observing a strong interest in developing rigorous techniques to make sound inference from biased, nonprobabilistic samples. For example, Wang et al. (2015)[37] showed that it is feasible to forecast election using data from surveys run on the videogame Xbox, if an appropriate approach that involves post-stratification is used.

Re-purposing data vs Re-purposing methods. Although there has always been data collected for goals other than research, today the mere scale of data that are available to anyone is so large that it is driving new directions of research. Repurposing data might become the norm in social sciences and it may lead to the development of new methods, as well as re-purposing classical approaches to the new "Big Data" context.

\section{Here comes the Data Revolution}

The potential emergence of a fourth paradigm, has not gone completely unnoticed within the demographic research community. The International Union for the Scientific Study of Population (IUSSP) has joined the movement initiated by the United Nations towards a Data Revolution, i.e. a "new international initiative to improve the 
quality of statistics and information available to citizens" [1] ${ }^{4}$. We shortly address two aspects of this Data Revolution: "new" old Big Data and the so-called digital breadcrumbs.

\section{1 "New" old Big Data}

Ruggles [30] describes an "explosion" in the availability of "Big Microdata" for population research. The approach pushed by Ruggles and his colleagues at IPUMS, with a strong basis at the University of Minnesota, is to make micro-level population data from censuses and other sources as accessible as possible for other researchers. These data should allow unprecedented opportunities for population research over time and place, with rich geographical detail.

Other examples on how old big demographic data can be used in a new way use innovative approaches, including crowd-sourcing, to extract information from paper-based demographic documents, including hand-written ones [11]. In this case, the meeting between demographers and computer scientists has been fruitful and is promising in potentially delivering a wide range of (big) micro data about several sources.

\subsection{Digital breadcrumbs}

\subsubsection{Re-purposing data}

The global spread of Internet and digital technologies, as well as the rapid diffusion of smartphones, have profoundly transformed our lives. As a consequence of the digital revolution, individuals leave an increasing quantity of traces online that can be analyzed to advance knowledge on population processes. Here we include examples of research that leveraged online data to study the three main components of demographic change: fertility, mortality and migration.

Fertility. Web searches represent the main online data source that has been used to study fertility. Reis and Brownstein (2010) show that the volume of Internet searches for abortion is inversely proportional to local abortion rates and directly proportional to local restrictions on abortion [28]. Billari et al. (2013) show that Google searches for fertility-related queries, like 'pregnancy' or 'birth', can be used to predict fertility intentions and fertility rates several months ahead [4]. Ojala et al. (2017) use Google Correlate to detect evidence for different socio-economic contexts related to fertility (e.g., teen fertility, fertility of high income households, etc.)[26] One of the most important messages of this line of literature is that combining traditional data sources with new data, like Web searches, can improve the predictive power of

${ }^{4}$ The two authors of this paper are also co-chairing the IUSSP Scientific Panel on "Big Data and Population Processes" established for the 2015-18 period. 
demographic models. However, that cannot be done in a naive way as correlations between aggregate Web searches and individual intentions may not persist for long periods of time. For example, the widely known Google flu approach to track influenza symptoms and detect potential outbreaks using Web searches [12] has been very useful and successful. However, at times, it also produced largely erroneous estimates, typically when the nature of the relationship between searches, news and behaviors changed [20]. Thus the results of these models have to be interpreted carefully and with caution.

Mortality. In the context of mortality analysis, the main source of online data results from decentralized collaborations that have produced genealogical data sets. For example, Fire and Elovici [8] use data collected from the WikiTree website to study correlations in lifespans among parents and children, as well as spouses. Similarly, Kaplanis et al. (2017) [16] leverage the data produced by enthusiasts of genealogy to evaluate population genetics theories on the dispersion of families. The key here is that (a) there are digital records that are left behind by people or institutions and (b) there is a critical mass of people who organize the data in meaningful ways for their own purposes and common goals.

Migration. Trends in international migrant flows have been estimated by tracking the locations, inferred from IP addresses, of users who repeatedly login into a Web service (e.g., Yahoo! [39, 34]). Geo-located Twitter tweets have been used to integrate the dimensions of internal and international migration [38] and to study global mobility patterns [13]. LinkedIn data have proven useful to evaluate trends in migration by educational attainment and sector of employment [33]. Google+ data, which provide pseudo migration histories, have proven useful to study how migrants connect countries within a network of flows. [23]

\subsubsection{Re-purposing methods}

Data science is about data, including re-purposing data. However, above all it is about the scientific use of data to advance knowledge. In this section we include a couple of examples of applications of classic social science methods and research design to the new data environment.

Demographic calibration. Non-representative digital breadcrumbs have to be calibrated against 'ground truth' data in order to evaluate biases and model them. Zagheni and Weber develop a method that combines the parsimonious perspective of model life tables, based on level and shape parameters, with standard calibration techniques [39]. The approach is inspired by calibration models for stochastic microsimulations [31]. The underlying idea in the microsimulation literature is that simulations may generate estimates of quantities of interest that are biased. Identifying and modeling the bias is thus key to make statistical inference. We can consider social media and the Internet as "laboratories" that produce estimates of quantities of interest that are biased, but in a systematic way. Here, "systematic", means that there are hidden, potentially stochastic rules that determine the relationship between the online data and the offline quantities of interest. Conditional on a model for the 
bias, statistical inference for the quantities of interest can be made usingtechniques like the Bayesian melding [27, 31].

Difference-in-differences. In some situations, "ground truth" data do not exist. Without any knowledge about the size and the direction of the bias, providing a reliable picture for the quantity of interest at one point in time is not possible. In these cases, instead of estimating the absolute value of variables of interest, a more modest task can be accomplished: estimating relative changes in quantities. This can be done using a difference-in-differences approach. A first demographic example includes estimating trends in migration patterns using geo-located Twitter data. [38] A second type of application relates to the evaluation of how shocks, like antiimmigrant laws, shape public sentiments about migration. [10]

\subsubsection{Can formal demography make a comeback?}

Can digital breadcrumbs offer new opportunities for formal demography? Online users form populations that can be analyzed using classic tools of formal demographic analysis. In turn, new types of population dynamics generate new questions that require new ways of formalization. Here we offer an illustrative example.

Consider users of a social media platform, like Twitter. The date when customers sign up for the service can be interpreted as a birth. The date when they stop tweeting for a long-enough interval of time can be interpreted as a death.

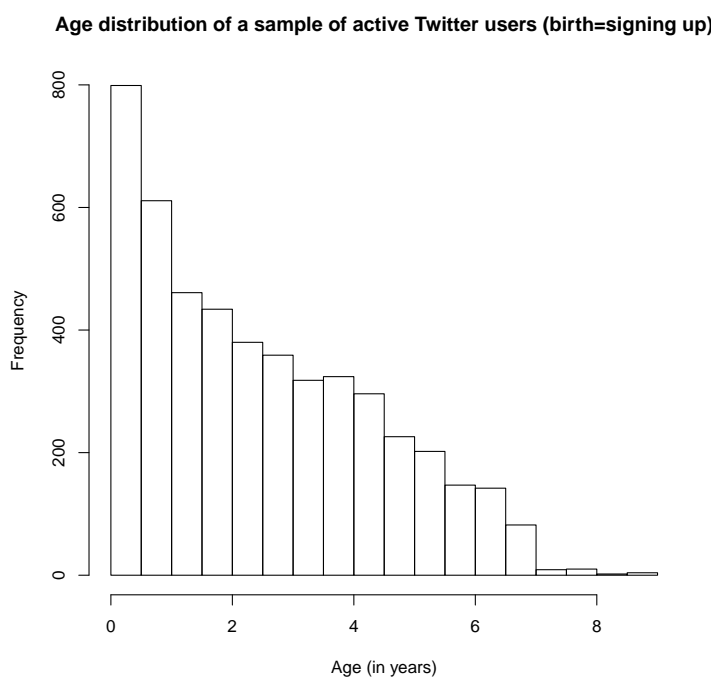

Fig. 4 Age distribution of a sample of active Twitter users (mid-2016), where 'birth' indicates the date when the user signed up. Source: own elaboration of data collected using the Twitter API. 
Figure 4 shows the age structure of a sample of active Twitter users (mid-2016). The histogram, which is equivalent to a population pyramid, reveals an age structure tilted towards 'young' users. In other words, it is a population that is growing rapidly. With these data only, we cannot say whether the growth in the population of Twitter users is driven by bots or real users, or whether it is related to 'life course transitions' of users, who may be quite active when they sign up, but then stop tweeting after a certain interval of time. However, we can use standard demographic techniques to estimate an approximate rate of growth in Twitter customers.

The problem can be stated as follows: given the number of individuals $P_{x}$ at age $x$ and $P_{y}$ at age $y$, at time $t$, the goal is to find the rate at which the births were increasing between years $t-x$ and $t-y$. It turns out that, under the assumption of exponential growth of births, the population rate of growth $r$ is (see Keyfitz and Caswell [18]):

$$
r=\frac{1}{y-x} \log \left(\frac{P_{x}}{P_{y}} \frac{L_{y}}{L_{x}}\right)
$$

where $L_{x}$ and $L_{y}$ are the fraction of people surviving $x$ and $y$ years, respectively.

For the illustrative example based on a small sample of Twitter users, one obtains an estimated annual growth rate of the Twitter population equal to around 0.3. This is extremely fast growth, compared to rates for human populations.

\section{Not a conclusion: The Data Revolution is not a dinner party}

If we believe that a paradigm shift in the study of population processes, around the emergence of "Big Data", is undergoing, it is by definition impossible to make firm conclusions. For sure, this "Data Revolution" will not be a dinner party.

Conventional wisdom will need to be challenged. Existing borders between disciplines might become a hindrance to scientific progress. Sticking to traditional approaches within the demographic research community might prevent further progress, or just let other, bolder, communities of scholars bring the advances needed to further our understanding of population processes. These challenges will need to be accompanied by new types of training for the younger generations of scholars-and perhaps even more relevantly, for the older generations. A fruitful way ahead is perhaps to combine traditional approaches with new one: counting and now-casting, indirect estimation and the used of non-representative Web-based data, official statistics and digital breadcrumbs.

A bit of patience, despite the speed of the field, is needed. Setbacks will happen and mistakes will be made within the "Data Revolution". Trial and errors are needed. Taking a very conservative stance that requires a new paradigm to have fully shown its potential in order to legitimize its approaches would however be an even bigger mistake. For the study of population processes, the Data Revolution is already here. 
Acknowledgements This project has received funding from the European Research Council (ERC) under the European Union's Horizon 2020 research and innovation programme (grant agreement n. 694262), project DisCont - Discontinuities in Household and Family Formation.

\section{References}

1. The IUSSP on a Data Revolution for development. Population and Development Review, 41(1):172-177, 2015.

2. Jakub Bijak, Daniel Courgeau, Eric Silverman, and Robert Franck. Quantifying paradigm change in demography. Demographic Research, 30:911-924, 2014.

3. Jakub Bijak, Jason Hilton, Eric Silverman, and Viet Dung Cao. Reforging the wedding ring: Exploring a semi-artificial model of population for the united kingdom with gaussian process emulators. Demographic Research, 29:729, 2013.

4. Francesco Billari, Francesco D'Amuri, and Juri Marcucci. Forecasting births using Google. In Annual Meeting of the Population Association of America, 2013.

5. Francesco C. Billari. Integrating macro-and micro-level approaches in the explanation of population change. Population Studies, 69(sup1):S11-S20, 2015.

6. Daniel Courgeau and Robert Franck. Demography, a fully formed science or a science in the making? an outline programme. Population-E, 62(01):39-45, 2007.

7. D. R. Cox. Regression models and life-tables. Journal of the Royal Statistical Society. Series B (Methodological), 34(2):187-220, 1972.

8. Michael Fire and Yuval Elovici. Data mining of online genealogy datasets for revealing lifespan patterns in human population. ACM Transactions on Intelligent Systems and Technology (TIST), 6(2):28, 2015.

9. Michel Fleury and Louis Henry. Des registres paroissiaux à l'histoire de la population: manuel de dépouillement et d'exploitation de l'état-civil ancien. Institut National d'Études Démographiques, Paris, 1956.

10. René D Flores. Do anti-immigrant laws shape public sentiment?: A study of Arizona's SB 1070 using Twitter data. American Journal of Sociology, 2017.

11. Alicia Fornés, Josep Lladós, Joan Mas, Joana Maria Pujades, and Anna Cabré. A bimodal crowdsourcing platform for demographic historical manuscripts. In Proceedings of the First International Conference on Digital Access to Textual Cultural Heritage, pages 103-108. ACM, 2014.

12. Jeremy Ginsberg, Matthew H Mohebbi, Rajan S Patel, Lynnette Brammer, Mark S Smolinski, and Larry Brilliant. Detecting influenza epidemics using search engine query data. Nature, 457(7232):1012-1014, 2008

13. Bartosz Hawelka, Izabela Sitko, Euro Beinat, Stanislav Sobolevsky, Pavlos Kazakopoulos, and Carlo Ratti. Geo-located Twitter as proxy for global mobility patterns. Cartography and Geographic Information Science, 41, 2014.

14. François Héran. The vocabulary of demography, from its origins to the present day: A digital exploration. Population-E, 70(3):497-536, 2015.

15. Tony Hey, Stewart Tansley, and Kristin M. Tolle. The fourth paradigm: data-intensive scientific discovery, volume 1. Microsoft Research, Redmond, WA, 2009.

16. Joanna Kaplanis, Assaf Gordon, Mary Wahl, Michael Gershovits, Barak Markus, Mona Sheikh, Melissa Gymrek, Gaurav Bhatia, Daniel G MarArthur, Alkes Price, et al. Quantitative analysis of population-scale family trees using millions of relatives. bioRxiv, page 106427, 2017.

17. Ridhi Kashyap and Francisco Villavicencio. The dynamics of son preference, technology diffusion, and fertility decline underlying distorted sex ratios at birth: A simulation approach. Demography, 53(5):1261-1281, 2016.

18. Nathan Keyfitz and Hal Caswell. Applied Mathematical Demography, volume 47. Springer, 2005. 
19. Thomas S. Kuhn. The Structure of Scientific Revolutions. Second Edition, Enlarged. University of Chicago Press, Chicago, IL, 1970.

20. David M Lazer, Ryan Kennedy, Gary King, and Alessandro Vespignani. The parable of Google Flu: Traps in big data analysis. Science, 2014.

21. Ronald Lee. Demography abandons its core, 2001.

22. Torkild Hovde Lyngstad and Torbjørn Skardhamar. Nordic register data and their untapped potential for criminological knowledge. Crime and Justice, 40(1):613-645, 2011.

23. Johnnatan Messias, Fabricio Benevenuto, Ingmar Weber, and Emilio Zagheni. From migration corridors to clusters: The value of Google+ data for migration studies. In Advances in Social Networks Analysis and Mining (ASONAM), 2016 IEEE/ACM International Conference on, pages 421-428. IEEE, 2016.

24. Tom Moultrie, Rob Dorrington, Allan Hill, Kenneth Hill, Ian Timæus, and Basia Zaba. Tools for Demographic Estimation. IUSSP, Paris, 2013.

25. Máire Ní Bhrolcháin and Tim Dyson. On causation in demography: Issues and illustrations. Population and Development Review, 33(1):1-36, 2007.

26. Jussi Ojala, Emilio Zagheni, Francesco C Billari, and Ingmar Weber. Fertility and its meaning: Evidence from search behavior. Proceedings of the International Conference on Web and Social Media (ICWSM) 2017, 2017.

27. David Poole and Adrian E Raftery. Inference for deterministic simulation models: the Bayesian melding approach. Journal of the American Statistical Association, 95(452):1244$1255,2000$.

28. Ben Y Reis and John S Brownstein. Measuring the impact of health policies using Internet search patterns: the case of abortion. BMC public health, 10(1):514, 2010.

29. Peter H. Rossi, James D. Wright, and Andy B. Anderson. Sample surveys: History, current practice, and future prospects. In Peter H. Rossi, James D. Wright, and Andy B. Anderson, editors, Handbook of Survey Research, chapter 1, pages 1-20. Academic Press, New York, NY, 1983.

30. Steven Ruggles. Big microdata for population research. Demography, 51(1):287-297, 2014.

31. Hana Ševčíková, Adrian E Raftery, and Paul A Waddell. Assessing uncertainty in urban simulations using Bayesian melding. Transportation Research Part B: Methodological, 41(6):652669, 2007.

32. J. Timothy Sprehe. The World Fertility Survey: An international program of fertility research. Studies in Family Planning, 5(2):35-41, 1974.

33. Bogdan State, Mario Rodriguez, Dirk Helbing, and Emilio Zagheni. Migration of professionals to the us: Evidence from LinkedIn data. In Proceedings of the 6th International Conference on Social Informatics (SocInfo), 2014.

34. Bogdan State, Ingmar Weber, and Emilio Zagheni. Studying inter-national mobility through IP geolocation. In WSDM, pages 265-274, 2013.

35. Richard Swedberg. The art of social theory. Princeton University Press, Princeton, NJ, 2014.

36. Andres Vikat, Zsolt Spéder, Gijs Beets, Francesco C Billari, Christoph Bühler, Aline Désesquelles, Tineke Fokkema, Jan M Hoem, Alphonse MacDonald, Gerda Neyer, et al. Generations and Gender Survey (GGS): Towards a better understanding of relationships and processes in the life course. Demographic research, 17:389-440, 2008

37. Wei Wang, David Rothschild, Sharad Goel, and Andrew Gelman. Forecasting elections with non-representative polls. International Journal of Forecasting, 31(3):980-991, 2015.

38. Emilio Zagheni, Venkata Rama Kiran Garimella, Ingmar Weber, et al. Inferring international and internal migration patterns from Twitter data. In Proceedings of the companion publication of the 23rd international conference on World wide web companion, pages 439-444. International World Wide Web Conferences Steering Committee, 2014.

39. Emilio Zagheni and Ingmar Weber. You are where you e-mail: using e-mail data to estimate international migration rates. In WebSci, pages 348-351, 2012. 